\title{
Comparación agronómica entre tipos de pepino (Cucumis sativus)
}

\section{José Aníbal Cruz-Coronado ${ }^{1}$ (D) , José Eladio Monge-Pérez ${ }^{2}$ iD $\&$ Michelle Loría Coto ${ }^{3}$ (iD}

1. Universidad de Costa Rica, San José, Costa Rica; cruzcsanibal@gmail.com

2. Universidad de Costa Rica, Estación Experimental Agrícola Fabio Baudrit Moreno, Alajuela, Costa Rica; melonescr@yahoo.com.mx

3. Universidad Estatal a Distancia, Escuela de Ciencias Exactas y Naturales, Sabanilla, Costa Rica; michelle_loria@yahoo.com

Recibido 23-IX-2019 • Corregido 7-II-2020 • Aceptado 25-II-2020

DOI: https://doi.org/10.22458/urj.v12i1.2842

\begin{abstract}
Agronomic comparison among cucumber (Cucumis sativus) types". Introduction: Cucumber is an important vegetable crop in Costa Rica. Objective: To evaluate yield and quality of three cucumber types (long, medium, and small) grown under greenhouse and hydroponic conditions. Methods: The crop was planted on coconut fiber as substrate, pruned to one stem per plant, and managed with fertigation at the Agricultural Experimental Station Fabio Baudrit Moreno located in Alajuela, Costa Rica.. Results: The harvest of small cucumber type started 26 days after transplant (dat), and for medium and long type cucumbers it started between 28 and 30dat. Data show a wide variability between cucumber types with respect to: fruit length $(11,89-$ $31,74 \mathrm{~cm})$, diameter $(43,15-50,37 \mathrm{~mm})$ and weight $(143,07-488,19 g)$; number of fruits $(22,81-90,14)$ and yield per plant $(8656,32-12911,67 \mathrm{~g})$; yield per area ( $\left.22,48-33,54 \mathrm{~kg} / \mathrm{m}^{2}\right)$; and percentage of total soluble solids $\left(3,03-3,22^{\circ} \mathrm{Brix}\right)$. The highest total number of fruits per plant $(90,14)$ resulted from small cucumber type, while the lowest number of fruits per plant $(22,81)$ and the fruits with the highest fruit weight $(488,19 \mathrm{~g})$ were obtained with long cucumber type. The total $\left(33,54 \mathrm{~kg} / \mathrm{m}^{2}\right)$, commercial $\left(27,10 \mathrm{~kg} / \mathrm{m}^{2}\right)$ and first quality yield $\left(16,41 \mathrm{~kg} / \mathrm{m}^{2}\right)$ were significantly higher with small cucumber type. Conclusions: Small cucumber type is considered the most recommended under the conditions tested from an economical point of view, although the consumer preference in Costa Rica for this cucumber type remains to be evaluated.
\end{abstract}

Keywords: Cucumis sativus, greenhouse, fruit length, yield, fruit weight, quality.
RESUMEN. Introducción: El pepino es un cultivo hortícola importante en Costa Rica. Objetivo: Evaluar el rendimiento y la calidad de tres tipos de pepino (largo, mediano y pequeño), cultivados bajo invernadero en condiciones hidropónicas. Métodos: El cultivo se manejó con fertirrigación, en sustrato de fibra de coco, y con poda a un tallo por planta en la Estación Experimental Agrícola Fabio Baudrit Moreno, en Alajuela, Costa Rica. Resultados: El pepino pequeño inició cosecha a los 26 días después del trasplante (ddt), mientras que los tipos mediano y largo lo hicieron entre los 28 y $30 \mathrm{ddt}$. Los datos muestran una amplia variabilidad entre los tipos de pepino en cuanto a: longitud $(11,89-31,74 \mathrm{~cm})$, diámetro $(43,15-50,37 \mathrm{~mm})$ y peso del fruto $(143,07-488,19 \mathrm{~g})$; número de frutos $(22,81-90,14)$ y rendimiento por planta $(8656,32$ - 12911,67g); rendimiento por área $(22,48$ $\left.33,54 \mathrm{~kg} / \mathrm{m}^{2}\right)$; y porcentaje de sólidos solubles totales $\left(3,03-3,22^{\circ} \mathrm{Brix}\right)$. El mayor número total de frutos por planta $(90,14)$ se obtuvo con el pepino pequeño, mientras que el menor número total de frutos por planta $(22,81)$ y los frutos con el mayor peso $(488,19 \mathrm{~g})$ se obtuvieron con el pepino largo. El mayor rendimiento total $\left(33,54 \mathrm{~kg} / \mathrm{m}^{2}\right)$, comercial $\left(27,10 \mathrm{~kg} / \mathrm{m}^{2}\right)$ y de primera calidad $\left(16,41 \mathrm{~kg} / \mathrm{m}^{2}\right)$ se obtuvo con el pepino pequeño. Conclusiones: Desde un punto de vista económico, se considera que el pepino pequeño es el más recomendable bajo las condiciones en que se desarrolló el ensayo, aunque está pendiente de evaluación su aceptación por parte del consumidor en Costa Rica

Palabras clave: Cucumis sativus, invernadero, longitud del fruto, rendimiento, peso del fruto, calidad 
El pepino, Cucumis sativus L., es una planta de la familia Cucurbitaceae, y es una hortaliza que se cultiva en condiciones tropicales y subtropicales alrededor del mundo (Kapuriya et al., 2017). Sus frutos se consideran una buena fuente de minerales y vitaminas (Sarhan \& Ismael, 2014).

El pepino posee varios cultivares en el mercado, con diferente tamaño, forma y coloración de los frutos, textura de la cáscara, sabor, y características vegetativas (Crosby, 2008). Una de las clasificaciones utiliza como criterio el origen, como es el caso de los tipos holandés y francés (también llamados europeos), y el tipo asiático. Otro criterio de clasificación es el tamaño del fruto: largo (tipo holandés), mediano (tipo americano o "slicer", y francés), y pequeño (tipo Beit Alpha, mini, o pepinillo).

Los pepinos híbridos tipo Beit-Alfa son relativamente delgados, de cáscara lisa y sin espinas, de color verde claro uniforme, y se cosechan entre 8 a $13 \mathrm{~cm}$; producen varios frutos por nudo, lo que aumenta el rendimiento de frutos, al compararlo con los tipos holandés que solo producen un fruto por nudo (Crosby, 2008); se utilizan para invernaderos, y al igual que el tipo holandés, tienen la cáscara delgada, y se deben proteger de los insectos y la deshidratación (Johnny's Selected Seeds, 2014).

Los pepinos tipo holandés son de sabor suave, sin semillas, y se cosechan de 30 a $36 \mathrm{~cm}$ de longitud; su cáscara es delgada, lisa y sin espinas, con una excelente calidad comestible, por lo que no requieren de pelado para su consumo (Crosby, 2008); son cultivados principalmente en invernadero (Johnny's Selected Seeds, 2014; Grijalva, Macías, Grijalva, \& Robles, 2011).

Los pepinos tipos americano ("slicer") son de color oscuro y cáscara gruesa, lo que hace que tengan un buen comportamiento poscosecha; se cosechan de 18 a $23 \mathrm{~cm}$ de longitud (Crosby, 2008; Johnny's Selected Seeds, 2014). Y los pepinos tipo asiático son espinosos, muy largos, y requieren tutorado para mantener los frutos rectos (Johnny's Selected Seeds, 2014).

El objetivo de esta investigación fue evaluar el rendimiento y la calidad de tres tipos de pepino (largo, mediano y pequeño), cultivados bajo ambiente protegido en condiciones hidropónicas, en Alajuela, Costa Rica.

\section{MATERIALES Y MÉTODOS}

Se sembraron 13 genotipos híbridos de pepino (Cucumis sativus) partenocárpico, correspondientes a tres tipos, según la longitud del fruto: largo, mediano y pequeño (Cuadro 1); el cultivo se realizó en condiciones hidropónicas, en el invernadero de Hortalizas de la Estación Experimental Agrícola Fabio Baudrit Moreno (EEAFBM), ubicada en Barrio San José de Alajuela, Costa Rica, a una altitud de 883msnm. El invernadero es modelo XR, marca Richel (Francia), tipo multicapilla, de plástico, con ventilación cenital automática.

El almácigo se sembró el 3 de julio de 2015, en bandejas plásticas rellenas con fibra de coco; y el trasplante se realizó el 15 de julio de 2015, cuando las plántulas tenían una hoja verdadera. El período de cultivo abarcó hasta el 20 de octubre de 2015, es decir, hasta los 97 días después del trasplante (ddt), para un período de cosecha de 10 semanas.

El cultivo se realizó en sacos plásticos rellenos con fibra de coco, de $1 \mathrm{~m}$ de largo, $20 \mathrm{~cm}$ de ancho y $15 \mathrm{~cm}$ de altura. La distancia de siembra fue de $25 \mathrm{~cm}$ entre plantas, y de $1,54 \mathrm{~m}$ entre hileras, para una densidad de 2,60plantas $/ \mathrm{m}^{2}$. Las plantas se manejaron a un solo tallo, eliminando todos los tallos secundarios. Se eliminaron los primeros cuatro frutos de cada planta, con el fin de lograr una cosecha más uniforme. El plan de fertirriego se presenta en el Cuadro 2. 


\section{CUADRO 1}

Genotipos de pepino evaluados

\begin{tabular}{ccc}
\hline Tipo de pepino & Genotipo & Empresa \\
\hline \multirow{2}{*}{ Largo } & Arioso & Known You Seed \\
$(\mathrm{n}=4)$ & Cumlaude & Rijk Zwaan \\
& Kalunga & Enza Zaden \\
& Paisaje & Rijk Zwaan \\
\hline & Corinto & Enza Zaden \\
& Katrina & Enza Zaden \\
Mediano & Macario & Enza Zaden \\
$(\mathrm{n}=7)$ & Modan & Rijk Zwaan \\
& Paraíso & Enza Zaden \\
& Primavera & Enza Zaden \\
& $22-20-782$ & Pandia Seeds \\
\hline \multirow{2}{*}{ Pequeño $(n=2)$} & Larino & Rijk Zwaan \\
& $22-20-781$ & Pandia Seeds \\
\hline
\end{tabular}

CUADRO 2

Plan de fertirriego utilizado

\begin{tabular}{|c|c|c|c|c|c|c|c|c|c|c|c|c|}
\hline \multirow[b]{2}{*}{ Etapa del cultivo } & \multicolumn{12}{|c|}{ Concentración del nutriente (mg/l) } \\
\hline & $\mathbf{N}$ & $\mathbf{P}$ & $\mathbf{K}$ & $\mathrm{Ca}$ & $\mathrm{Mg}$ & $\mathbf{S}$ & $\mathrm{Cu}$ & $\mathrm{Fe}$ & $\mathrm{Zn}$ & Mn & Mo & B \\
\hline $0-15 \mathrm{ddt}$ & 150 & 53 & 240 & 165 & 40 & 50 & 0,16 & 2,9 & 0,3 & 0,6 & 0,9 & 0,8 \\
\hline $15-30 \mathrm{ddt}$ & 161 & 53 & 265 & 175 & 50 & 50 & 0,16 & 2,9 & 0,3 & 0,6 & 0,9 & 0,8 \\
\hline $30 \mathrm{ddt}$ en adelante & 172,5 & 53 & 290 & 175 & 55 & 50 & 0,16 & 2,9 & 0,3 & 0,6 & 0,9 & 0,8 \\
\hline
\end{tabular}

Se clasificó la cosecha según categorías de calidad (Cuadro 3). Se consideró como rendimiento comercial la suma de las categorías de primera y segunda calidad, y como rendimiento total la suma de las tres categorías de calidad.

\section{CUADRO 3}

Parámetros de calidad de pepino utilizados en el ensayo

\begin{tabular}{cccc}
\hline & \multicolumn{3}{c}{ Categoría de calidad } \\
\cline { 2 - 4 } Parámetro & Primera & Segunda & Rechazo \\
\hline Deformación de frutos & Ausente & Leve & Severa \\
Daños en la cáscara del & Ausente & Menor o igual a $1 \mathrm{~cm}^{2}$ & Mayor a $1 \mathrm{~cm}^{2}$ \\
\hline
\end{tabular}

Se evaluaron las siguientes variables:

1. Longitud del fruto $(\mathrm{cm})$ : Se midió esta característica en 20 frutos de cada categoría de calidad, y se obtuvo el promedio.

2. Diámetro del fruto $(\mathrm{mm})$ : Se midió esta característica en la parte media de 20 frutos de cada categoría de calidad, y se obtuvo el promedio.

3. Edad al inicio de la cosecha (ddt): Se contabilizó el número de días transcurridos desde el trasplante hasta la fecha del primer corte de frutos.

4. Número de frutos por planta: Se contabilizó el número total de frutos producidos por parcela, y se dividió entre el número de plantas de la parcela.

5. Peso del fruto (g): Se midió el peso total de los frutos producidos por categoría de calidad en cada parcela, y se dividió entre el número total de frutos obtenidos.

6. Rendimiento por planta (g/planta): Se midió el peso total de la producción en cada parcela, y se dividió entre el número de plantas por parcela.

7. Rendimiento por área $\left(\mathrm{kg} / \mathrm{m}^{2}\right)$ : Se calculó a partir del rendimiento por planta y de la densidad de siembra. 
8. Porcentaje de sólidos solubles totales ( $\left.{ }^{\circ} B r i x\right)$ : Se evaluó esta característica a partir de un macerado de la pulpa y la placenta del fruto, en 20 frutos de cada categoría de calidad, y se obtuvo el promedio.

El peso de los frutos se determinó por medio de una balanza electrónica marca Ocony, modelo UWE HGM-20, con una capacidad de $20000 \pm 1 \mathrm{~g}$. Para obtener la longitud del fruto se utilizó una cinta métrica marca Assist, modelo 32G-8025, con una capacidad de 800,0 $\pm 0,1 \mathrm{~cm}$. El diámetro de los frutos se determinó con un calibrador digital marca Mitutoyo, modelo CD-6"CS, con una capacidad de 15,00 $\pm 0,01 \mathrm{~cm}$. El porcentaje de sólidos solubles totales se midió con un refractómetro manual marca Boeco, con una capacidad de 0,0 $-32,0 \pm 0,2^{\circ}$ Brix.

Se utilizó un diseño experimental irrestricto al azar, donde los tratamientos fueron cada tipo de pepino, y cada genotipo correspondió a una repetición. Para cada genotipo se sembró una parcela con ocho plantas (dos sacos), y todos los datos se obtuvieron a partir de los frutos totales producidos en dicha parcela. Para todas las variables se realizó un análisis estadístico de variancia, y se utilizó la prueba de LSD Fisher con una significancia de $5 \%$ para determinar diferencias entre los tratamientos.

Ética, conflicto de intereses y declaración de financiamiento: El autor declara haber cumplido con todos los requisitos éticos y legales pertinentes, tanto durante el estudio como en el manuscrito; que no hay conflictos de interés de ningún tipo, y que todas las fuentes financieras se detallan plena y claramente en la sección de agradecimientos. Asimismo, está de acuerdo con la versión editada final del documento. El respectivo documento legal firmado se encuentra en los archivos de la revista.

\section{RESULTADOS}

Durante el ensayo, las condiciones ambientales dentro del invernadero fueron las siguientes: la temperatura diurna promedio fue de $27,1^{\circ} \mathrm{C}$, con un valor mínimo promedio de $20,7^{\circ} \mathrm{C}$, y un máximo promedio de $33,4^{\circ} \mathrm{C}$; mientras que la temperatura nocturna promedio fue de $21,4^{\circ} \mathrm{C}$, con un valor mínimo promedio de $19,8^{\circ} \mathrm{C}$ y un máximo promedio de $23,9^{\circ} \mathrm{C}$. La humedad relativa promedio durante el día fue de $72,3 \%$, y durante la noche fue de $89,2 \%$. La radiación fotosintéticamente activa diurna promedio fue de $494,0 \mathrm{~W} / \mathrm{m}^{2}$.

Con respecto al inicio de la producción de frutos, los pepinos tipo pequeño iniciaron su cosecha a los $26 \mathrm{ddt}$, mientras que los tipo mediano y largo lo hicieron entre los $28 \mathrm{y} 30 \mathrm{ddt}$.

En el Cuadro 4 se presentan los datos para la longitud del fruto. Al considerar los datos totales, lógicamente los frutos de pepino largo mostraron una longitud significativamente mayor $(31,74 \mathrm{~cm})$ en comparación a los otros dos tipos de pepino, e igualmente el pepino mediano mostró un valor significativamente mayor $(19,84 \mathrm{~cm})$ para esta característica en comparación con el pepino pequeño $(11,89 \mathrm{~cm})$; esto se presentó también para las tres categorías de calidad.

Se encontraron diferencias significativas en el diámetro del fruto (Cuadro 5). Al considerar el total de frutos, el pepino pequeño presentó un diámetro del fruto significativamente menor $(43,15 \mathrm{~mm})$ que los pepinos largo y mediano (entre 50,01 y $50,37 \mathrm{~mm}$ ), y entre estos dos tipos de pepino no se presentaron diferencias significativas. El pepino pequeño también presentó los frutos con el menor diámetro en todas las categorías de calidad. Sin embargo, para los frutos de primera calidad, el pepino mediano mostró un diámetro del fruto significativamente mayor en comparación con el pepino largo. 


\section{CUADRO 4}

Longitud del fruto $(\mathrm{cm})$, según tipo de pepino

\begin{tabular}{ccccc}
\hline & \multicolumn{4}{c}{ Longitud del fruto $(\mathbf{c m})$, según categoría de calidad } \\
\cline { 2 - 5 } Tipo de pepino & Total & Primera & Segunda & Rechazo \\
\hline Largo & $31,74 \mathrm{c}$ & $33,37 \mathrm{c}$ & $31,21 \mathrm{c}$ & $30,64 \mathrm{c}$ \\
Mediano & $19,84 \mathrm{~b}$ & $21,13 \mathrm{~b}$ & $19,53 \mathrm{~b}$ & $18,85 \mathrm{~b}$ \\
Pequeño & $11,89 \mathrm{a}$ & $12,59 \mathrm{a}$ & $12,00 \mathrm{a}$ & $11,09 \mathrm{a}$ \\
\hline *Valores con una letra en común no son significativamente diferentes, según prueba LSD Fisher (p $\leq 0,05)$.
\end{tabular}

\section{CUADRO 5}

Diámetro del fruto $(\mathrm{mm})$, según tipo de pepino

\begin{tabular}{ccccc}
\hline & \multicolumn{4}{c}{ Diámetro del fruto $(\mathbf{m m})$, según categoría de calidad } \\
\cline { 2 - 5 } Tipo de pepino & Total & Primera & Segunda & Rechazo \\
\hline Largo & $50,01 \mathrm{~b}$ & $51,21 \mathrm{~b}$ & $49,80 \mathrm{~b}$ & $49,01 \mathrm{~b}$ \\
Mediano & $50,37 \mathrm{~b}$ & $53,47 \mathrm{c}$ & $50,14 \mathrm{~b}$ & $47,50 \mathrm{~b}$ \\
Pequeño & $43,15 \mathrm{a}$ & $47,30 \mathrm{a}$ & $43,32 \mathrm{a}$ & $38,85 \mathrm{a}$ \\
\hline *Valores con una letra en común no son significativamente diferentes, según prueba LSD Fisher (p $\leq 0,05)$.
\end{tabular}

En el Cuadro 6 se muestran los datos para el número de frutos por planta. Al considerar la totalidad de frutos, el pepino pequeño produjo una cantidad de frutos por planta significativamente superior $(90,14)$ en comparación con los pepinos largo y mediano; esto también sucedió para todas las categorías de calidad. El pepino mediano produjo una cantidad de frutos por planta significativamente mayor para el total de frutos producidos $(34,50)$, en comparación al pepino largo $(22,81)$; y lo mismo aconteció para todas las categorías de calidad.

\section{CUADRO 6}

Número de frutos por planta, según tipo de pepino

\begin{tabular}{ccccc}
\hline & \multicolumn{4}{c}{ Número de frutos por planta, según categoría de calidad } \\
\cline { 2 - 5 } Tipo de pepino & Total & Primera & Segunda & Rechazo \\
\hline Largo & $22,81 \mathrm{a}$ & $7,75 \mathrm{a}$ & $7,80 \mathrm{a}$ & $7,26 \mathrm{a}$ \\
Mediano & $34,50 \mathrm{~b}$ & $13,28 \mathrm{~b}$ & $11,95 \mathrm{~b}$ & $9,27 \mathrm{~b}$ \\
Pequeño & $90,14 \mathrm{C}$ & $38,32 \mathrm{c}$ & $29,19 \mathrm{c}$ & $22,64 \mathrm{c}$ \\
\hline * Valores con una letra en común no son significativamente diferentes, según prueba LSD Fisher (p $\leq 0,05)$.
\end{tabular}

En el Cuadro 7 se muestran los datos de peso del fruto para los tipos de pepino evaluados. Al considerar la producción total, como era previsible, el pepino largo obtuvo frutos con un peso significativamente superior $(488,19 \mathrm{~g})$ con respecto a los otros tipos de pepino; y el pepino mediano presentó un peso del fruto significativamente superior $(254,29 \mathrm{~g})$ con respecto al pepino pequeño $(143,07 \mathrm{~g})$; esto se presentó también en todas las categorías de calidad.

En el Cuadro 8 se presentan los datos de rendimiento por planta para los tipos de pepino evaluados. Al considerar el total de frutos producidos, el pepino pequeño produjo un rendimiento significativamente superior (12911,67g/planta) con respecto a los otros tipos de pepino, y el pepino largo produjo un rendimiento mayor (11 153,04g/planta) con respecto al pepino mediano (8 $656,32 \mathrm{~g} /$ planta). Además, al considerar la categoría de primera calidad, el pepino pequeño produjo un rendimiento por planta significativamente mayor (6 318,46g/planta) en comparación con los otros dos tipos de pepino (entre 3 763,36 y 3 928,28g/planta).

En el Cuadro 9 se presentan los datos obtenidos en el rendimiento por área para los diferentes tipos de pepino. El pepino pequeño obtuvo un rendimiento total $\left(33,54 \mathrm{~kg} / \mathrm{m}^{2}\right)$, comercial $\left(27,10 \mathrm{~kg} / \mathrm{m}^{2}\right)$ y de primera calidad $\left(16,41 \mathrm{~kg} / \mathrm{m}^{2}\right)$ significativamente superior, en comparación con los otros tipos de pepino evaluados. Por lo tanto, desde un punto de vista económico, el pepino tipo 
pequeño se considera el más recomendable (dado el mayor precio de los frutos de primera calidad), bajo las condiciones en que se desarrolló el ensayo.

\section{CUADRO 7}

Peso del fruto $(\mathrm{g})$, según tipo de pepino

\begin{tabular}{ccccc}
\hline & \multicolumn{4}{c}{ Peso del fruto (g), según categoría de calidad } \\
\cline { 2 - 5 } Tipo de pepino & Total & Primera & Segunda & Rechazo \\
\hline Largo & $488,19 \mathrm{c}$ & $508,26 \mathrm{c}$ & $481,85 \mathrm{c}$ & $473,39 \mathrm{c}$ \\
Mediano & $254,29 \mathrm{~b}$ & $291,23 \mathrm{~b}$ & $241,57 \mathrm{~b}$ & $220,94 \mathrm{~b}$ \\
Pequeño & $143,07 \mathrm{a}$ & $164,57 \mathrm{a}$ & $140,71 \mathrm{a}$ & $109,52 \mathrm{a}$ \\
\hline
\end{tabular}

*Valores con una letra en común no son significativamente diferentes, según prueba LSD Fisher $(p \leq 0,05)$.

\section{CUADRO 8}

Rendimiento por planta (g/planta), según tipo de pepino

\begin{tabular}{ccccc}
\hline & \multicolumn{4}{c}{ Rendimiento por planta (g/planta), según categoría de calidad } \\
\cline { 2 - 5 } Tipo de pepino & Total & Primera & Segunda & Rechazo \\
\hline Largo & $11153,04 \mathrm{~b}$ & $3928,28 \mathrm{a}$ & $3764,60 \mathrm{~b}$ & $3460,16 \mathrm{c}$ \\
Mediano & $8656,32 \mathrm{a}$ & $3763,36 \mathrm{a}$ & $2880,71 \mathrm{a}$ & $2012,26 \mathrm{a}$ \\
Pequeño & $12911,67 \mathrm{c}$ & $6318,46 \mathrm{~b}$ & $4115,52 \mathrm{~b}$ & $2477,71 \mathrm{~b}$ \\
\hline *Valores con una letra en común no son significativamente diferentes, según prueba LSD Fisher (p $\leq 0,05)$.
\end{tabular}

En el Cuadro 10 se presentan los valores de porcentaje de sólidos solubles totales de los tres tipos de pepino evaluados. Al considerar el total de frutos producidos, los pepinos tipo pequeño y mediano presentaron un valor significativamente menor (entre 3,03 y 3,05 ${ }^{\circ} \mathrm{Brix}$ ) para esta característica, en comparación con el pepino largo $\left(3,22^{\circ}\right.$ Brix); lo mismo sucedió en los frutos de primera y de segunda calidad.

\section{CUADRO 9}

Rendimiento por área $(\mathrm{kg} / \mathrm{m} 2)$, según tipo de pepino

\begin{tabular}{cccccc}
\hline & \multicolumn{5}{c}{ Rendimiento $\left(\mathbf{k g} / \mathbf{m}^{2}\right)$, según categoría de calidad } \\
\cline { 2 - 5 } Tipo de pepino & Total & Comercial & Primera & Segunda & Rechazo \\
\hline Largo & $28,97 \mathrm{~b}$ & $19,98 \mathrm{~b}$ & $10,20 \mathrm{a}$ & $9,78 \mathrm{~b}$ & $8,99 \mathrm{c}$ \\
Mediano & $22,48 \mathrm{a}$ & $17,26 \mathrm{a}$ & $9,78 \mathrm{a}$ & $7,48 \mathrm{a}$ & $5,23 \mathrm{a}$ \\
Pequeño & $33,54 \mathrm{c}$ & $27,10 \mathrm{c}$ & $16,41 \mathrm{~b}$ & $10,69 \mathrm{~b}$ & $6,44 \mathrm{~b}$ \\
\hline *Valores con una letra en común no son significativamente diferentes, según prueba LSD Fisher $(\mathrm{p} \leq 0,05)$.
\end{tabular}

\section{CUADRO 10}

Porcentaje de sólidos solubles totales ('Brix), según tipo de pepino.

\begin{tabular}{ccccc}
\hline & \multicolumn{4}{c}{ Porcentaje de sólidos solubles totales ( ${ }^{\circ}$ Brix), según categoría de calidad } \\
\cline { 2 - 5 } Tipo de pepino & Total & Primera & Segunda & Rechazo \\
\hline Largo & $3,22 \mathrm{~b}$ & $3,29 \mathrm{~b}$ & $3,28 \mathrm{~b}$ & $3,10 \mathrm{~b}$ \\
Mediano & $3,03 \mathrm{a}$ & $3,08 \mathrm{a}$ & $3,03 \mathrm{a}$ & $2,99 \mathrm{a}$ \\
Pequeño & $3,05 \mathrm{a}$ & $3,08 \mathrm{a}$ & $3,01 \mathrm{a}$ & $3,05 \mathrm{ab}$ \\
\hline *Valores con una letra en común no son significativamente diferentes, según prueba LSD Fisher $(\mathrm{p} \leq 0,05)$.
\end{tabular}




\section{DISCUSIÓN}

Con respecto a la edad de la planta al inicio de la cosecha, según varios investigadores, el inicio de la cosecha oscila entre 31 y 91ddt para pepino largo; entre 31 y $90 \mathrm{ddt}$ para pepino mediano; y entre 24 y 59ddt para pepino pequeño (ver Cuadro 11, Apéndice Digital); en comparación con esta información, los resultados del presente ensayo se encontraron dentro de ese ámbito únicamente para el pepino pequeño, pero fueron menores a dichos ámbitos en el caso del pepino largo y el mediano, es decir, que estos tipos de pepino mostraron una producción más precoz. Esta precocidad puede haber sido inducida por la alta temperatura y radiación que se presentaron en el ensayo, lo cual habría ocasionado un aceleramiento en el metabolismo de las plantas, pues una mayor temperatura acelera el crecimiento de la planta y la maduración del fruto (Grijalva et al., 2011).

Según diversos autores, la longitud del fruto varía entre 25,91 y $39,30 \mathrm{~cm}$ para pepino largo; entre 21,10 y $26,55 \mathrm{~cm}$ para pepino mediano; y entre 12,43 y $21,90 \mathrm{~cm}$ para pepino pequeño (Cuadro 12. Apéndice Digital); los resultados (datos totales) para el pepino tipo largo en la presente investigación se ubicaron dentro de dicho ámbito, pero en el caso de los pepinos tipo mediano y pequeño, los valores fueron menores a los informados en la literatura.

En relación al diámetro del fruto, varios investigadores han indicado que los valores oscilan entre 43,0 y $53,3 \mathrm{~mm}$ para pepino largo; entre 24,2 y $60,7 \mathrm{~mm}$ para pepino mediano; y entre 24,4 y $58,0 \mathrm{~mm}$ para pepino pequeño (Cuadro 13, Apéndice Digital); los resultados del presente ensayo se encontraron dentro de dichos ámbitos.

De acuerdo con diversos autores, la producción total de frutos varía entre 8,0 y 24,0 frutos/planta para pepino largo; entre 8,00 y 24,31 frutos/planta para pepino mediano; y entre 6,0 y 66,8 frutos/planta para pepino pequeño (Cuadro 14, Apéndice Digital); los resultados de la presente investigación se ubicaron dentro de dichos ámbitos únicamente para el pepino largo, mientras que en el caso de los pepinos tipo mediano y pequeño se superaron ampliamente dichos ámbitos, lo que indica una mayor prolificidad.

La presencia de altas temperaturas $\left(>30^{\circ} \mathrm{C}\right.$ ) durante la producción de pepino provoca desequilibrios en las plantas, dando lugar a malformaciones de hojas y frutos defectuosos (Grijalva et al., 2011). Esto probablemente se presentó en el presente ensayo, dado que las temperaturas máximas dentro del invernadero alcanzaron inclusive los $41^{\circ} \mathrm{C}$, lo que pudo haber colaborado con la producción de muchos frutos de calidad de rechazo, en especial en el pepino tipo largo, en el cual el 37,9\% de los frutos correspondió a dicha categoría de calidad, mientras que estos valores fueron menores para el pepino mediano $(24,7 \%)$ y el pequeño $(27,5 \%)$.

Según varios investigadores, el peso del fruto oscila entre 278,0 y 616,90 g para pepino largo; entre 103,7 y 415,66g para pepino mediano; y entre 44,0 y 330,00g para pepino pequeño (Cuadro 15, Apéndice Digital); los resultados del presente ensayo se encontraron dentro de dichos ámbitos.

Con relación al rendimiento por planta, diversos autores han informado que varía entre 576,9 y $11600 \mathrm{~g} /$ planta para pepino largo; entre 1424 y 13 409g/planta para pepino mediano; y entre 810 y $13800 \mathrm{~g} /$ planta para pepino pequeño (Cuadro 16, Apéndice Digital); los resultados de la presente investigación se ubicaron dentro de dichos ámbitos.

De acuerdo con varias investigaciones, el rendimiento por área oscila entre 1,8 y $25,90 \mathrm{~kg} / \mathrm{m}^{2}$ para pepino largo; entre 6,00 y $27,33 \mathrm{~kg} / \mathrm{m}^{2}$ para pepino mediano; y entre 2,38 y $25,25 \mathrm{~kg} / \mathrm{m}^{2}$ para pepino pequeño (Cuadro 17, Apéndice Digital); los resultados del presente ensayo se encontraron dentro de dichos ámbitos únicamente para el pepino mediano, mientras que en el caso de los pepinos tipo largo y pequeño se obtuvo un valor muy superior al ámbito informado, es decir, que fueron mucho más productivos. 
Según diversos autores, el porcentaje de sólidos solubles totales varía entre 3,38 y $3,67^{\circ} \mathrm{Brix}$ para pepino largo; entre 3,00 y $4,08^{\circ}$ Brix para pepino mediano; y de entre 2,5 y $5,0^{\circ}$ Brix para pepino pequeño (Cuadro 18, Apéndice Digital); los resultados de la presente investigación se ubicaron dentro de dichos ámbitos para los pepinos tipo mediano y pequeño, pero en el caso del pepino largo el valor obtenido fue inferior a dicho ámbito.

El mayor número total de frutos por planta $(90,14)$ se obtuvo con el pepino tipo pequeño, mientras que el menor número total de frutos por planta $(22,81)$ y los frutos con el mayor peso $(488,19 \mathrm{~g})$ se obtuvieron con el pepino tipo largo.

Se concluye que el mayor rendimiento total $\left(33,54 \mathrm{~kg} / \mathrm{m}^{2}\right)$, comercial $\left(27,10 \mathrm{~kg} / \mathrm{m}^{2}\right)$ y de primera calidad $\left(16,41 \mathrm{~kg} / \mathrm{m}^{2}\right)$ se obtuvo con el pepino pequeño, por lo que, desde un punto de vista económico, se considera el más recomendable bajo las condiciones en que se desarrolló el ensayo, aunque está pendiente de evaluación su aceptación por parte del consumidor en el mercado costarricense.

\section{AGRADECIMIENTOS}

Los autores agradecen el financiamiento recibido por parte de CONARE, así como de la Universidad de Costa Rica, para la realización de este trabajo. Asimismo, agradecen la colaboración de Mario Monge en la revisión de la traducción del resumen al idioma inglés.

\section{REFERENCIAS}

Crosby, L. C. (2008). Growth and consumer evaluation of Cucumis sativus L. cultivated in controlled environments. (Tesis de maestría en Horticultura). Texas Tech University, Texas, EEUU.

Grijalva, R. L., Macías, R., Grijalva, S. A., \& Robles, F. (2011). Evaluación del efecto de la fecha de siembra en la productividad y calidad de híbridos de pepino europeo bajo condiciones de invernadero en el noroeste de Sonora. Biotecnia, 13(1), 29-36.

Johnny's Selected Seeds. (2014). Cucumber types and terminology. Recuperado de http://www.johnnyseeds.com/assets/information/cucumbers-types-terminology-8989.pdf

Kapuriya, V. K., Ameta, K. D., Teli, S. K., Chittora, A., Gathala, S., \& Yadav, S. (2017). Effect of spacing and training on growth and yield of polyhouse grown cucumber (Cucumis sativus L.). International Journal of Current Microbiology and Applied Sciences, 6(8), 299-304.

Sarhan, T. Z., \& Ismael, S. F. (2014). Effect of low temperature and seaweed extracts on flowering and yield of two cucumber cultivars (Cucumis sativus L.). International Journal of Agricultural and Food Research, 3(1), 41-54. 